\title{
Maternal smoking during pregnancy affects adult onset of asthma in offspring: a follow up from birth to age 46 years
}

\author{
Sanna Toppila-Salmi ${ }^{1,2}$, Annika T. Luukkainen ${ }^{1}$, Baizhuang $\mathrm{Xu}^{3}$, Jussi Lampi ${ }^{3}$, \\ Juha Auvinen ${ }^{4}$, Kishor Dhaygude ${ }^{1}$, Marjo-Riitta Järvelin ${ }^{4,5}$ and Juha Pekkanen $\mathbb{1}^{3,6}$ \\ Affiliations: ${ }^{1}$ Medicum, Haartman Institute, University of Helsinki, Helsinki, Finland. ${ }^{2}$ Skin and Allergy \\ Hospital, Helsinki University Hospital, Helsinki, Finland. ${ }^{3}$ Environment Health Unit, National Institute for \\ Health and Welfare, Kuopio, Finland. ${ }^{4}$ Center for Life Course Health Research, Oulu, Finland. ${ }^{5}$ mperial \\ College London, London, UK. 'Dep of Public Health, University of Helsinki, Helsinki, Finland.
}

Correspondence: Sanna Toppila-Salmi, Skin and Allergy Hospital, Helsinki University Hospital, P.0. Box 160, 00029 HUS, Finland. E-mail: sanna.salmiahelsinki.fi

@ERSpublications

Maternal smoking during pregnancy is linked to cumulative incidence of asthma in offspring between 31 and 46 years. The association was accentuated in those reporting at age 31 as having past respiratory problems and/or who had haplotype rs11702779-AA. http://bit.ly/2WgR91H

Cite this article as: Toppila-Salmi S, Luukkainen $\mathrm{AT}, \mathrm{Xu} \mathrm{B}$, et al. Maternal smoking during pregnancy affects adult onset of asthma in offspring: a follow up from birth to age 46 years. Eur Respir J 2020; 55: 1901857 [https://doi.org/10.1183/13993003.01857-2019].

\section{ABSTRACT}

Rationale: Environmental tobacco smoke (ETS) exposure increases asthma risk in children. There is limited knowledge of prenatal ETS for adult-onset asthma.

Objectives: To determine the association between prenatal ETS and adult onset asthma.

Measurements and main results: The questionnaire and clinical data of 5200 people, free of physiciandiagnosed asthma by 31 years of age, who were included in the Northern Finland Birth Cohort 1966 Study was used. The association of maternal smoking during the last 3 months of pregnancy with onset of physician-diagnosed asthma and with lung function in adult offspring was studied using adjusted multivariate regression analyses. The cumulative incidence of physician-diagnosed asthma between the ages of 31 and 46 years was $5.1 \%$ among men and $8.8 \%$ among women. Gestational smoke exposure was associated with adult-onset asthma among offspring (adjusted OR 1.54, 95\% CI 1.04-2.29), namely among offspring who reported either past non-diagnosed asthma (OR 9.63, 95\% CI 2.28-40.67) or past cough with wheeze $(3.21,95 \%$ CI 1.71-6.05). A significant association was detected between gestational smoke exposure and the offspring's forced expiratory volume in $1 \mathrm{~s}\left(\mathrm{FEV}_{1}\right) /$ forced vital capacity (FVC) ratio at 31 years of age. In offspring with the haplotype rs11702779-AA of RUNX1, gestational smoke exposure was associated with adult-onset asthma (5.53, 95\% CI 2.11-14.52, adjusted p-value for interaction 0.10$)$. Conclusion: Maternal smoking during pregnancy is associated with the cumulative incidence of asthma in offspring between the ages of 31 and 46 years. The association was accentuated in offspring who at age 31, reported having past respiratory problems and/or who had haplotype rs11702779-AA. A reduction in $\mathrm{FEV}_{1} / \mathrm{FVC}$ ratio was also observed at age 31 years in offspring with gestational smoke exposure. These results could reflect the early vulnerability of offspring's airways to ETS and its putative long-term effects.

This article has supplementary material available from erj.ersjournals.com

Received: 20 Sept 2019 | Accepted after revision: 4 March 2020

Copyright OERS 2020 


\section{Introduction}

Smoking during pregnancy is common, with an estimated prevalence of $\leqslant 38 \%$ in Europe [1], and is the single largest modifiable risk for all pregnancy-related morbidity and mortality [2-6]. Studies have shown that gestational environmental tobacco smoke (ETS) exposure increases the risk of wheezing in childhood or asthma among offspring during early childhood [7, 8], in preschool-age children [9], in adolescents [10, $11]$, and in adults $[12,13]$. Young maternal age at delivery and passive smoking in utero or during childhood have been documented to increase risk for adult-onset asthma [14, 15].

Genetic [16-21] and genomic data [22] have identified susceptibility genes affecting asthma risk due to tobacco smoke. These single-nucleotide polymorphisms (SNPs) are found in the loci of genes, such as genes encoding glutathione S-transferase (GSTM1, GSTT1, GSTP1), Gasdermin (GSDMB, GSDML), ORMDL3, the Sm protein family (LSM14A), protein tyrosine phosphatase receptor (PTPRT) and runt-related transcription factor 1 (RUNX1).

There is still limited knowledge as to whether gestational smoke exposure is associated with cumulative incidence of asthma or decreased lung function in adult offspring. The aim was to study the association between gestational smoke exposure and the incidence of asthma in adult offspring in the Northern Finland Birth Cohort with a follow-up of 46 years. We asked about the smoking habits of pregnant mothers and investigated the offspring's cumulative incidence of adult-onset asthma, and lung function. The hypothesis was that gestational smoke exposure is associated with the cumulative incidence of asthma at 46 years of age, and this risk is increased in those with a genetic predisposition and/or in those reporting past respiratory problems.

\section{Methods}

The study population was based on the Northern Finland Birth Cohort in 1966 and consisted of mothers and their children who were born in the northern Finland provinces of Oulu and Lapland in 1966 [23]. The total number of births was 12058 , which covered $96.3 \%$ of all births in the area (see supplementary material). The birth cohort data regarding the prenatal and perinatal environment were collected via questionnaires and clinical examinations during the mothers' clinical visit when pregnant. Follow-ups were conducted at the ages of 31 [24] and 46 years. At 46 years of age in 2012-2013, 10300 (85.4\%) subjects were alive and traced and were given the opportunity to respond to two questionnaires $[25,26]$. Of these subjects, 7148 (69\%) completed data collection, which accounted 59\% of the original birth cohort population. The study was approved by the Ethics Committee of the Finnish Institute of Occupational Health and by the Ethics Committee of the Northern Ostrobothnia Hospital District. At all stages of the study, the subjects gave written informed consent according to the Declaration of Helsinki.

Data on self-reported physician-diagnosed asthma and self-reported asthma at 46 years of age was collected using questionnaires in 2012-2013. To determine incident asthma between 31 and 46 years of age, we used the following questions at 31 years and at 46 years of age, framed to obtain the patient's own opinion and physician diagnosis: "Have you ever had the following symptoms and/or diseases that are associated with airways?" Q1 framed for own opinion: Asthma: Never, Yes during the past 12 months, Yes but only previously. Q2 framed for doctor diagnosis or treated: Asthma: No, Yes. Answer criteria were as follows:

1) No physician-diagnosed asthma at 31 years of age when responding at 31 years of age. Q2 "No" (or Q2 “Missing” and Q1 "No").

2) Physician-diagnosed asthma at 46 years of age when responding at 46 years of age. Q2 "Yes" (and Q1 "Yes, current" or "Yes, only previous" or "Missing").

3) No physician-diagnosed asthma at 46 years of age when responding at 46 years of age. Q2 "No" (or Q2 “Missing” and Q1 “No").

The inclusion criterion was that all subjects fulfilled point 1, no physician-diagnosed asthma at 31 years of age. The incident physician-diagnosed asthma was the outcome measurement and it was defined by either "Yes" ( point 2) physician-diagnosed asthma at 46 years of age or "No" ( point 3) no physician-diagnosed asthma at 46 years of age.

A total of 5961 subjects answered the questions at both 31 and 46 years of age. Of these subjects, 5491 met the inclusion criteria. Final analyses were limited to 5200 subjects, with complete information on both the mother's smoking status during the last 3 months of pregnancy and information on asthma at both 31 and 46 years.

Subjects who reported at 46 years of age having self-reported asthma not diagnosed by a doctor "during the past 12 months" were labelled "beginning asthma". Subjects who reported at 46 years of age having self-reported asthma not diagnosed by a doctor "but only previously" were labelled as "past asthma". 
Similarly, subjects who reported at 46 years of age having wheeze "during the past 12 months" were labelled "current wheeze", and subjects who reported at 46 years of age having had wheeze "only previously" were labelled as "past wheeze". Those who reported current/past wheeze/asthma without physician-diagnosed asthma (both shortened as "respiratory problems") at 31 years of age were included for evaluation of possible interactions.

\section{Information on maternal smoking during pregnancy and on covariates}

Information about maternal smoking during pregnancy was collected via a questionnaire sent to mothers between the 6th and 7th month of pregnancy. Mothers were regarded as smokers during pregnancy if they reported smoking $\geqslant 1$ cigarette/pipe per day during the last 3 months of pregnancy. Mothers were considered smokers before pregnancy if they reported smoking $\geqslant 1$ cigarette/pipe per day during the 12 months before pregnancy. Changes in smoking status during pregnancy was based on the responses: no; gave up; cut down; no change; increased. The two latter responses were pooled due to the small number. We excluded offspring whose mothers had illogically responded or had not responded to these questions during their pregnancy. Information on covariates was collected during the mother's clinical visits during pregnancy and delivery, and included: the newborn's Apgar score (health index) at the 1st min; ponderal index (birth weight $(\mathrm{g}) \times 100 /$ crown-heel length $\left.(\mathrm{cm})^{3}\right)$; parity (the number of times the mother had carried pregnancies to a viable gestational age); maternal education; and parental asthma. The subject's own information on education and smoking status at 31 years of age was collected via postal questionnaires during the 31-year follow-up in 1997.

\section{Spirometry data at 31 years of age}

Of the 5200 offspring included in the analyses, we obtained spirometry data from 3777 offspring at 31 years of age. Spirometry and the variables used have previously been described [27]. The variables used were forced expiratory volume in $1 \mathrm{~s}\left(\mathrm{FEV}_{1}\right)$, forced vital capacity $(\mathrm{FVC})$ and $\mathrm{FEV}_{1} / \mathrm{FVC}$.

\section{Genetic data at 31 years of age}

We used a set of SNPs previously identified in the literature as having a putative risk of asthma together with tobacco smoke exposure: GSTM1, GSTT1, GSTP1, ADRB2, IL3, GSDMB, GSDML-ORMDL3, GSDMB, LSM14A, PTPRT, RUNX1 (supplementary table S4). Blood samples of the subjects in this cohort were collected for genotyping at age 31 (i.e. in 1997) and genetic data was available for 5402 individuals. Genotyping was completed using Illumina HumanCNV370DUO Analysis BeadChip (Illumina Inc., San Diego, CA, USA) and the BeadStudio 3.1 (www.illumina.com) algorithm, as previously described [28]. The data of the set of SNPs was obtained from genome-wide association study (GWAS) data.

\section{Statistical analyses}

Differences in the distribution of selected variables were assessed in relation to maternal smoking during pregnancy. The associations of the maternal smoking variables, such as maternal smoking before pregnancy, during the last 3 months of pregnancy, the amount of smoking before pregnancy and smoking habit changes during pregnancy on development of new asthma between the ages of 31 and 46 years, were examined using Pearson's Chi-squared test. Because the four maternal smoking variables were highly correlated, only maternal smoking during the last 3 months of pregnancy was examined in multivariate analyses. This was done using binary logistic regression analysis adjusted for selected covariates: the mother's educational level, parental asthma, the offspring's educational level and smoking (at 31 years of age), the ponderal index (in tertile), the Apgar score at the 1st min and parity. Binary logistic regression was also used to test the effect modifiers of the association between maternal smoking and adult-onset asthma, and the associations between SNPs and adult-onset asthma adjusted for the same selected covariates as previously mentioned. The associations between maternal smoking and lung function values $\left(\mathrm{FEV}_{1}\right.$ and $\mathrm{FEV}_{1} / \mathrm{FVC}$ ratio) were studied in linear regression models adjusted for the same selected covariates as previously mentioned. Deviation from the Hardy-Weinberg equilibrium was evaluated using the linkage disequilibrium (LD) calculator (www.ensembl.org/Homo_sapiens/Tools/LD) and $\mathrm{r}^{2} \geqslant 0.7$ was considered as LD between SNPs. The biomaRt package in Bioconductor [29] was used for retrieval of all annotation data from ensemble gene and SNP databases for these input human genomic variants. The data for all attributes (e.g. gene symbols, chromosomal coordinates, rs id, allele, etc.) was filtered out based on the scope of the study. Finally, additional SNP annotation of these human genomic variants was carried out using ANNOVAR [30]. All annotation database files (e.g. hg19 gnomad_genome and snp138) were downloaded using the command line and information about minor allele frequency was retrieved for all variants (supplementary table S4). Rare variants (MAF for Finnish $<0.05$ ) and variants that were not available in the GWAS data were excluded from the analyses. All other analyses were performed using SPSS Base 18 Statistical Software Package (SPSS, Chicago, IL, USA). 
TABLE 1 The association of maternal smoking during the last 3 months of pregnancy with cumulative incidence of physician-diagnosed asthma among offspring between 31 and 46 years of age

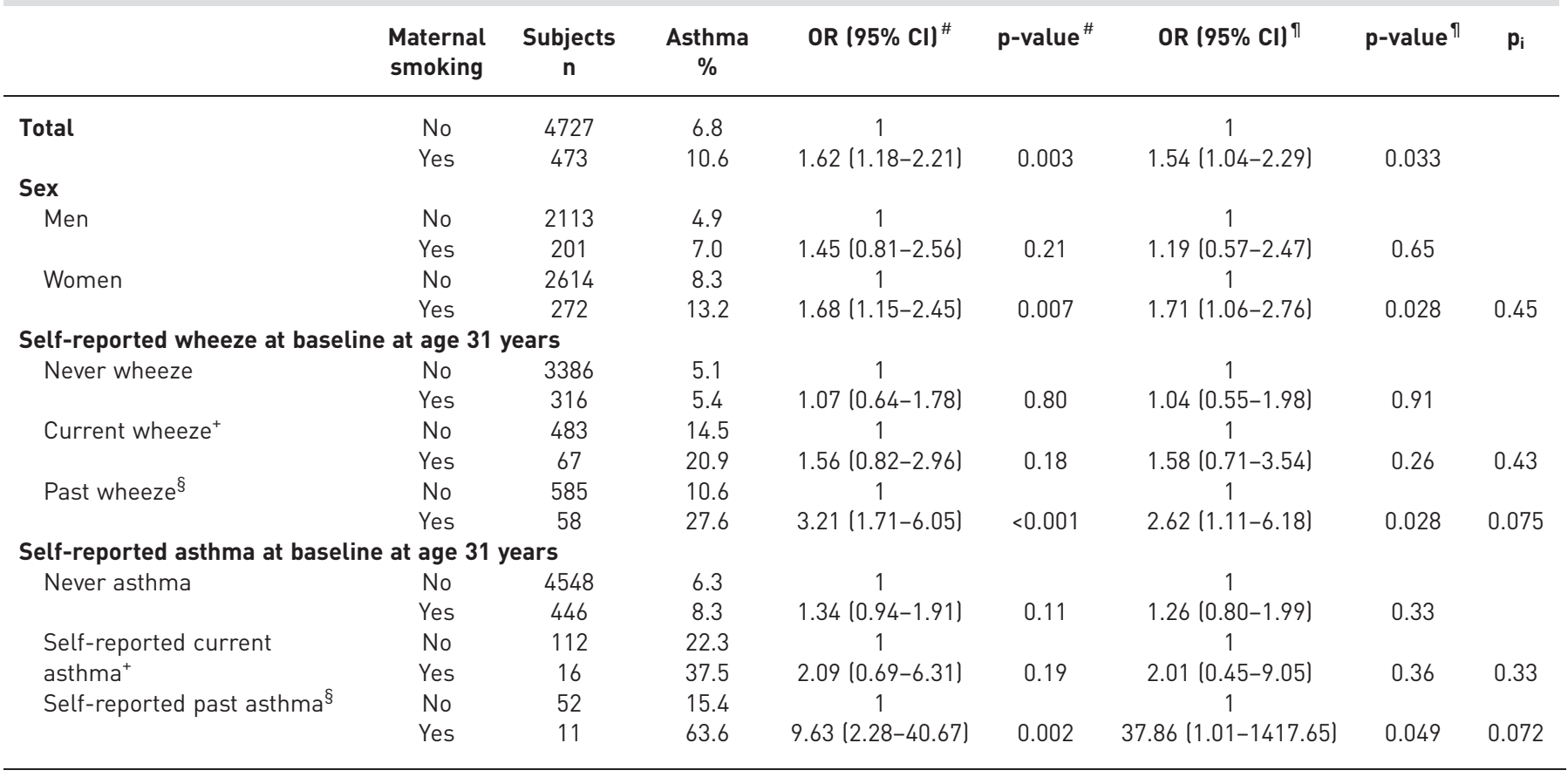

All subjects were free of physician-diagnosed asthma at age 31 years of age. $\mathrm{p}_{\mathrm{i}}$ : adjusted p-value for interaction ${ }^{\#}$ : crude; ${ }^{\text {?: }}$ adjusted for selected covariates (mother's education level, Apgar score at $1 \mathrm{~min}$, ponderal index at birth, parity, parental asthma, offspring's own education level at 31 years of age and offspring's smoking status at 31 years of age; ${ }^{+}$: during the last 12 months; ${ }^{\S}$ : not during the last 12 months, but earlier. The adjusted $\mathrm{p}_{\mathrm{i}}$ between maternal smoking and offspring smoking at 31 years of age was 0.32 .

\section{Results}

The proportion of mothers who smoked during the last 3 months of pregnancy was $8.6 \%$ (table 1). We observed 372 new physician-diagnosed asthma cases between 31 and 46 years of age among 5200 subjects who were free of an asthma diagnosis at the 31-year follow-up (table 1 and supplementary table S1). The cumulative incidence for new asthma diagnosis was $7.2 \%, 8.8 \%$ and $5.1 \%$, in all subjects, females and males, respectively. All maternal smoking variables associated statistically significantly with asthma onset between 31 and 46 years of age (table 1 and supplementary table S1). Mothers who either smoked 12 months before pregnancy or smoked during the last 3 months of pregnancy increased the risk for offspring's asthma in adulthood (supplementary table S1). The cumulative incidence of asthma was statistically insignificantly higher if mothers did not change their smoking habits or increased the amount smoked during the pregnancy, as compared with mothers who gave up smoking during pregnancy (supplementary table S1).

Maternal smoking variables correlated highly ( $\mathrm{p}=0.01, \mathrm{r}>0.63$ with Spearman rank correlation test). Therefore, maternal smoking during the last 3 months of pregnancy was selected for further multivariate analyses. A higher risk for asthma development between 3146 years of age was observed among offspring if mothers smoked at least one cigarette per day during the last 3 months of pregnancy (adjusted OR 1.54 (95\% CI 1.04-2.29) (table 1).

The cumulative incidence of asthma onset between 31 and 46 years of age was significantly higher in female offspring with gestational maternal smoking exposure during the last 3 months of pregnancy, compared with female offspring without gestational smoke exposure during the last 3 months of pregnancy (table 1 and supplementary table S1). A higher risk of asthma development between 31 and 46 years of age was observed among female offspring if mothers smoked at least one cigarette per day during the last 3 months of pregnancy (table 1 and supplementary table S1). The risk was independent of the covariates adjusted in the multivariate model (table 1). There was no interaction between sex and maternal smoking status during the last 3 months of pregnancy, on cumulative incidence of asthma between 31 and 46 years of age (adjusted p-value 0.45 ; table 1 ). 
Gestational smoke exposure and asthma onset in offspring with past respiratory problems Gestational maternal smoking exposure during the last 3 months of pregnancy was more strongly associated with offspring's adult-onset physician-diagnosed asthma in the offspring subgroup who reported current or past wheeze at baseline at 31 years of age, compared with offspring with no history of wheeze (table 1 and supplementary table S3). This difference reached statistical significance for past wheeze (adjusted p-value for interaction 0.075; table 1), but not for current wheeze (adjusted p-value for interaction 0.43; table 1). Similarly, gestational maternal smoking exposure during the last 3 months of pregnancy was more strongly associated with offspring's adult-onset physician-diagnosed asthma in the offspring subgroup, who reported self-reported non-diagnosed current or past asthma at 31 years of age, as compared with offspring with no history of asthma (table 1 and supplementary table S2). This difference reached statistical significance for past non-diagnosed asthma (adjusted p-value for interaction 0.072; table 1) but not for current non-diagnosed asthma at 31 years of age (adjusted p-value for interaction 0.33 ; table 1 ).

\section{Gestational smoke exposure and offspring lung function at 31 years of age}

Of the 5200 offspring included in the analyses, we had spirometry data for 3775 offspring at 31 years of age. Gestational maternal smoking exposure during the last 3 months of pregnancy was significantly associated with a reduced offspring $\mathrm{FEV}_{1} / \mathrm{FVC}$ ratio at 31 years of age (adjusted $\beta$-coefficient $-0.056, \mathrm{t}=$ $-3.24,95 \%$ CI $-0.020-0.005, \mathrm{p}=0.001$; table 2 ). When performing multivariate analysis using a linear regression model, the offspring's regular smoking at 31 years of age was also statistically significantly associated with the current $\mathrm{FEV}_{1} / \mathrm{FVC}$ ratio ( $\beta$-coefficient $-0.075, \mathrm{t}=-4.29,95 \%$ CI $-0.017--0.006$, $\mathrm{p}<0.001$ ), whereas other potential confounding factors did not have a significant association. Gestational maternal smoking exposure during the last 3 months of pregnancy was not significantly associated with the offspring's $\mathrm{FEV}_{1}$ value at 31 years of age (table 2). Those in the lowest $\mathrm{FEV}_{1}$ value quintile of at 31 years of age had an increased cumulative incidence of asthma between 31 and 46 years of age (OR 1.59, 95\% CI 1.21-2.10, $\mathrm{p}=0.001$, adjusted for offspring's sex, height and weight) compared with the highest quintile. When adjusted for maternal smoking, maternal education level, ponderal index, Apgar score at the 1st min, parity, parental asthma, the offspring's education and smoking at 31 years of age, the result remained similar (adjusted OR $1.4895 \%$ CI 1.10-2.0, p=0.011). Those in the lowest quintile of $\mathrm{FEV}_{1} / \mathrm{FVC}$ ratio at 31 years of age were associated with a cumulative incidence of asthma between 31 and 46 years of age (OR 1.71, 95\% CI 1.27-2.30, p<0.001; adjusted OR 1.64, 95\% CI 1.19-2.26, p=0.003). The $\mathrm{FEV}_{1} / \mathrm{FVC}$ ratio at 31 years of age was not an effect modifier in the association between gestational maternal smoking exposure during the last 3 months of pregnancy and the offspring's adult-onset physician-diagnosed asthma (adjusted p-value for interaction 0.88; table 2). Nor was the $\mathrm{FEV}_{1}$ value at 31 years of age an effect modifier in this association (adjusted p-value for interaction 0.99; table 2).

\section{The association of genetic predisposition and maternal smoking in offspring asthma and/or lung} function in adulthood

Six out of 12 SNPs of the following genes had MAF for Finnish $>5 \%$ and data available, and were entered in the analyses: ADRB2, IL3, GSDMB, LSM14A, RUNX1 (supplementary table S4). None of the haplotypes was enriched with certain living municipality of the offspring (data not shown). The SNPs rs8069176 and

TABLE 2 The association of maternal smoking during the last 3 months of pregnancy with spirometry values at 31 years of age

\begin{tabular}{|c|c|c|c|c|c|c|c|c|c|c|}
\hline & \multirow[t]{2}{*}{ Maternal smoking } & \multirow[t]{2}{*}{ Subjects $n$} & \multirow[t]{2}{*}{ Median } & \multirow[t]{2}{*}{ IQR } & \multicolumn{3}{|c|}{ Model $\#$} & \multicolumn{3}{|c|}{ Model ${ }^{\Uparrow}$} \\
\hline & & & & & $\boldsymbol{\beta}$ & $95 \% \mathrm{Cl}$ & $\mathrm{p}$-value & $\boldsymbol{\beta}$ & $95 \% \mathrm{Cl}$ & p-value \\
\hline $\mathrm{FEV}_{1}$ & No & 3434 & 3.85 & $3.35-4.54$ & Ref. & & & Ref. & & \\
\hline \multirow[t]{2}{*}{$\mathrm{FEV}_{1} / \mathrm{FVC}$} & No & 3434 & 0.85 & $0.81-0.88$ & Ref. & & & Ref. & & \\
\hline & Yes & 341 & 0.83 & $0.79-0.87$ & -0.072 & $-0.02--0.01$ & $<0.001$ & -0.056 & $-0.02--0.01$ & 0.001 \\
\hline
\end{tabular}

All subjects were free of physician-diagnosed asthma at 31 years of age. Spirometry data were available for 3775 offspring at 31 years of age. IQR: interquartile range (25th-75th percentile); $\beta$ : standardised $\beta$-coefficient; $F E V_{1}$ : forced expiratory volume in $1 \mathrm{~s} ; F E_{1} / F V C$ : ratio of $F E V_{1}$ and forced vital capacity. \#: adjusted according to the offspring's sex, height and weight at 31 years of age; ": adjusted according to the offspring's sex, height and weight at 31 years of age, and the mother's education level, the Apgar score at 1 min, the ponderal index at birth, parity, parental asthma, the offspring's own education level at 31 years of age and the offspring's smoking status at 31 years of age. $p$-values were

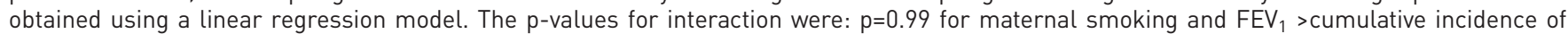
asthma between 31 and 46 years of age; $p=0.88$ for maternal smoking and $F E V_{1} / F V C$ ratio >cumulative incidence of asthma 31 and 46 years of age. p-values for interaction were adjusted according to the variables of Model ${ }^{\text {Il }}$ 
rs4795400 of the GSDMB gene were in LD $\left(\mathrm{r}^{2}=0.98, \mathrm{D}^{\prime}=1.00\right)$. The other SNPs were not in LD. In female offspring, rs1042713-GG of ADRB2 was associated with adult-onset asthma (adjusted OR 1.74, 95\% CI 1.06-2.84; $\mathrm{p}=0.028$ ). The SNPs of genes IL3, GSDMB, LSM14A and RUNX1 were not significantly associated with adult-onset asthma (data not shown). In female offspring who carried haplotype AA of RUNX1-gene, maternal smoke exposure was statistically significantly associated with adult-onset asthma, when adjusted according to sex, maternal education, ponderal index, Apgar score at the 1st min, parity, parental asthma, the offspring's education and smoking at 31 years of age $(\mathrm{p}=0.001$, adjusted $\mathrm{p}$-value for interaction 0.10; table 3). In female offspring, who also carried certain haplotypes of the genes LSM14A, $A D R B 2$, IL3 and GSDMB, maternal smoke exposure was statistically significantly associated with adult-onset asthma, yet no significant interaction was detected between haplotype and maternal smoke exposure (adjusted p-value for interaction $>0.3$; table 3 ). In male offspring, these phenomena were not detected (table 3). None of the SNPs were associated with the offspring's lung function parameters at 31 years of age (data not shown).

\section{Discussion}

This birth cohort study aimed to evaluate the association between gestational smoke exposure and the cumulative incidence of doctor-diagnosed asthma in offspring between 31 and 46 years of age. Subjects with past/current respiratory problems were analysed separately as it was hypothesised that they might interact with maternal smoke exposure on the cumulative incidence of asthma in adult offspring. Maternal smoking during pregnancy is associated with the cumulative incidence of asthma in adult offspring between 31 and 46 years of age. The association was accentuated in offspring who reported at age 31 as having past respiratory problems and/or who had haplotype rs11702779-AA. In addition, a reduction in the $\mathrm{FEV}_{1} / \mathrm{FVC}$ ratio was observed at 31 years of age in offspring with gestational smoke exposure.

Gestational smoke exposure was also associated with adult-onset asthma in offspring who carried haplotype rs11702779-AA of RUNX1. This association was slightly more prominent in female offspring. These observed relations were independent of several confounders, which reflect the prenatal and postnatal environment. RUNX1 encodes a transcription factor that regulates the differentiation of haematopoietic stem cells into mature blood cells and the development of the sensory neurons. SNPs in RUNX1 are associated with airway responsiveness in asthmatic children and these associations are modified by intra-uterine smoke exposure [17]. In line with this, we were able to detect interaction between the RUNX1 haplotype and maternal smoking exposure in offspring adult-onset asthma. LSM14A encodes the Sm protein family, a family of RNA-binding proteins which are expressed in every cellular organism and are related to pre-mRNA splicing [31, 32]. In 2017, a study showed that allele G of rs1759092 interacts with active tobacco smoking in adult-onset asthma [22]. In line with this, we detected that offspring who carried rs1759092-AG and who had maternal smoke exposure had a higher cumulative incidence of asthma in adulthood compared with other haplotypes. The pathomechanisms behind gestational exposure to maternal smoking and asthma onset at middle age are unclear. Grandmothers who smoked when pregnant with the mother increased the risk of persistent asthma in the grandchild independently of the mother's smoking status $[19,33,34]$, suggesting trans-generational epigenetic changes. It may therefore be possible that offspring with a genetic predisposition would have an increased adult asthma risk due to epigenetic reprogramming after gestational tobacco smoke exposure.

The relationship between gestational smoke exposure and childhood asthma development is well documented and presents a major health problem for generations to come [3, 7-11, 35]. Only a few follow-up studies have examined the effect of gene-environmental interactions on adult-onset of asthma $[36,37]$. A study in which parental smoking exposure was assessed retrospectively using a questionnaire to adult offspring documented that both intra-uterine exposure to maternal smoking during pregnancy and postnatal exposure to ETS adversely affected adult lung function and increased the risk of adult-onset asthma [12]. In studies in which parental smoke exposure was also retrospectively assessed using a questionnaire to adult offspring, cumulative and recent ETS exposure was shown to increase the risk of adult-onset asthma [38]. Heavy maternal smoking during childhood was shown to predispose to spirometrically defined COPD [39]. A European population-based study showed that early life factors, such as maternal age during delivery and maternal smoking, were significantly associated with $\mathrm{FEV}_{1}$ decline in adult offspring [40]. A three-generation study of a European population showed that the father's smoking during early adolescence and the grandmother's and mother's smoking during pregnancy may independently increase the offspring's risk of ever-asthma [41]. An Australian 50-year follow-up study of a childhood asthma population showed a synergistic impact of offspring smoking and asthma on lung function [36], which has also been documented in an adult population [42]. Maternal smoking seems to synergise with personal smoking by increasing airflow limitation in adults [15, 42]. A study of US children showed that prenatal and postnatal tobacco smoke exposure was not independently associated with airflow 
TABLE 3 The association of maternal smoking during the last 3 months of pregnancy with the cumulative incidence of physician-diagnosed asthma amongst offspring between 31 and 46 years years of age

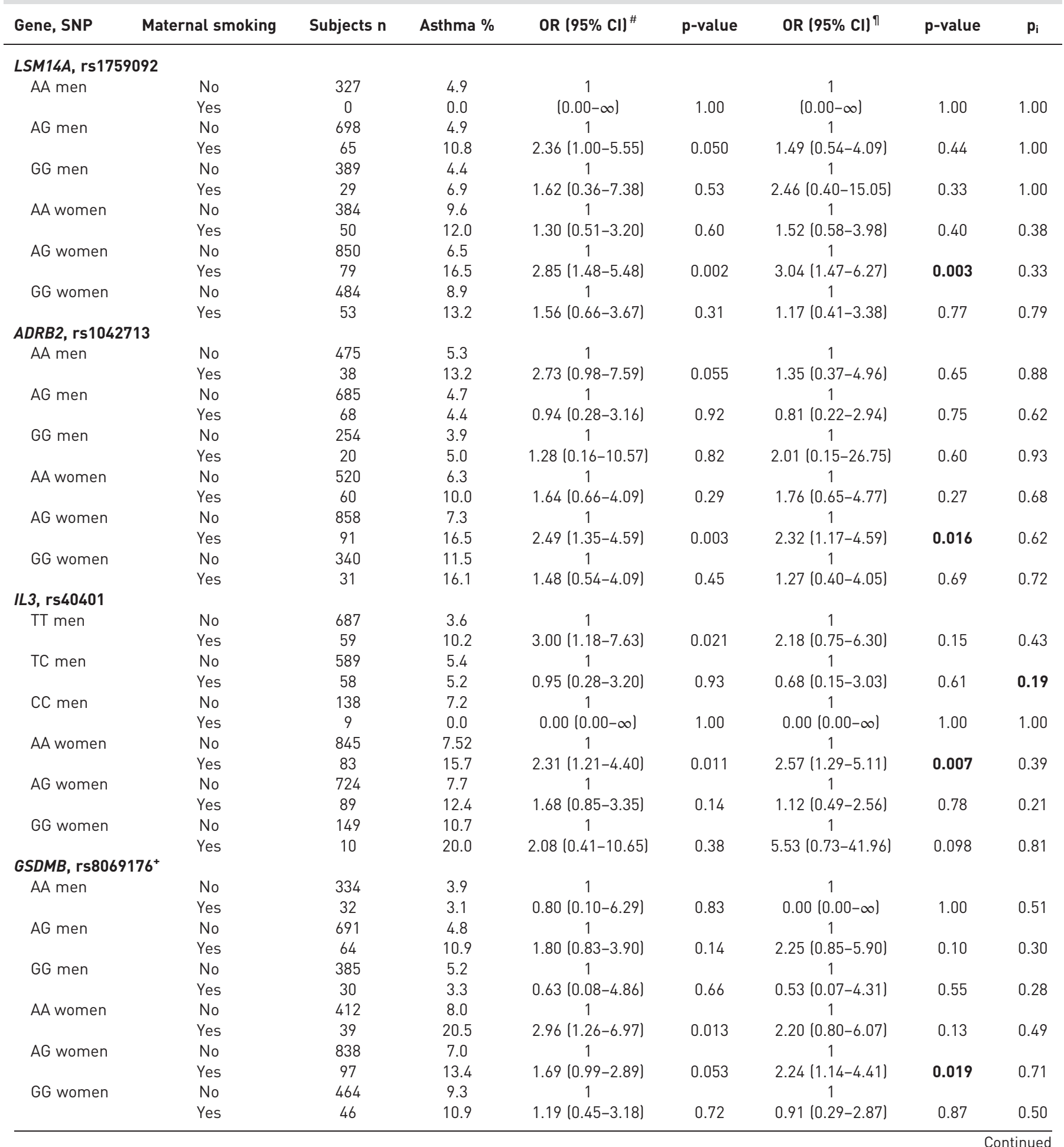


TABLE 3 Continued

\begin{tabular}{|c|c|c|c|c|c|c|c|c|}
\hline Gene, SNP & Maternal smoking & Subjects $\mathrm{n}$ & Asthma \% & OR $(95 \% \mathrm{CI})^{\#}$ & $\mathrm{p}$-value & OR $(95 \% \mathrm{CI})^{\uparrow}$ & p-value & $\mathbf{p}_{\mathrm{i}}$ \\
\hline TT men & No & 329 & 4.0 & 1 & & 1 & & \\
\hline \multirow[t]{2}{*}{ TC men } & No & 696 & 4.9 & 1 & & 1 & & \\
\hline & Yes & 63 & 11.1 & $2.43(1.03-5.74)$ & 0.042 & $2.17(0.82-5.67)$ & 0.12 & 1.00 \\
\hline CC men & No & 389 & 5.1 & 1 & & 1 & & \\
\hline AA women & Yes & 38 & 18.4 & $2.59(1.06-6.32)$ & 0.037 & $1.84(0.63-5.39)$ & 0.27 & 0.42 \\
\hline \multirow[t]{2}{*}{$A G$ women } & No & 838 & 7.0 & 1 & & 1 & & \\
\hline & Yes & 98 & 14.3 & $2.20(1.18-4.11)$ & 0.013 & $2.42(1.25-4.68)$ & 0.009 & 0.89 \\
\hline \multirow[t]{2}{*}{ GG women } & No & 469 & 9.2 & 1 & & 1 & & \\
\hline & Yes & 46 & 10.9 & $1.21(0.45-3.22)$ & 0.71 & $0.92(0.29-2.90)$ & 0.89 & 0.32 \\
\hline \multicolumn{9}{|c|}{ RUNX1, rs11702779 } \\
\hline GG men & Yes & 28 & 3.6 & $0.75(0.09-6.07)$ & 0.79 & $0.70(0.07-6.94)$ & 0.76 & 0.70 \\
\hline \multirow[t]{2}{*}{ AA women } & No & 572 & 6.3 & 1 & & 1 & & \\
\hline & Yes & 42 & 21.4 & $4.06(1.81-9.13)$ & 0.001 & $5.53(2.11-14.52)$ & 0.001 & 0.10 \\
\hline \multirow[t]{2}{*}{$A G$ women } & No & 771 & 8.6 & 1 & & 1 & & \\
\hline & Yes & 95 & 13.7 & $1.69(0.90-3.20)$ & 0.11 & 1.62 (0.79-3.29) & 0.19 & 0.089 \\
\hline \multirow[t]{2}{*}{ GG women } & No & 280 & 10.0 & 1 & & 1 & & \\
\hline & Yes & 33 & 9.1 & $0.90(0.26-3.14)$ & 0.87 & $0.98(0.27-3.64)$ & 0.98 & 0.054 \\
\hline
\end{tabular}

All subjects were free of physician-diagnosed asthma at 31 years of age. The models were stratified according to haplotype of the susceptibility genes and sex. $\mathrm{p}_{\mathrm{i}}$ : adjusted $\mathrm{p}$-value for interaction between gestational smoking exposure and gene haplotype in asthma risk; SNP: single-nucleotide polymorphism. " : crude; " : adjusted for selected covariates (mother's education level, Apgar score at 1 min, ponderal index at birth, parity, parental asthma, offspring's own education level at 31 years of age and offspring's smoking status at 31 years of age); ${ }^{+}$: SNPs that are in linkage disequilibrium lall the other SNPs were not in linkage disequilibrium). Values in bold have significance at the level of $p<0.05$ and $\mathrm{p}_{\mathrm{i}<} 0.2$.

obstruction in school-aged children; however, it was associated with airflow obstruction in children with asthma [43].

Controversial information exists as to whether smoking increases the risk of adult-onset asthma. There are studies that have either: failed to show an association [44, 45]; have not found an increased risk [46]; or that have found an association only in females [47]. There is evidence that parental smoking behaviour is associated with the offspring's smoking uptake risk [48]. Therefore, both parental smoking and inherited smoking behaviour may have an increased risk of adult-onset asthma among offspring. In our study, adjustment according to the offspring's reported smoking status at 31 years of age did not alter or did not have an interaction with the observed association between gestational smoke exposure and the cumulative incidence of asthma between 31 years and 46 years of age. This might be due to the low number of subjects.

The strengths of our study include the population-based design, data collection since pregnancy and a follow-up of 46 years. A further strength is that the detailed questionnaire on smoking habits carried out during pregnancy, minimising the effect of recall bias. Mothers who responded illogically to questions about nonsmoking and quitting smoking were not included in the study. We focused our study only on offspring who did not have doctor-diagnosed asthma before 31 years of age. Yet we allowed current or past self-reported respiratory problems at 31 years of age, in order to dissect out the offspring subgroup having putatively the highest risk of gestational smoking exposure to adult-onset asthma. This allowed us to demonstrate within 473 new adult asthma onsets that gestational smoke exposure directly predisposes to development of late-onset asthma in middle-aged offspring. This occurred in offspring reporting past respiratory problems, which is in line with previous observations on active or passive smoking [47-49].

This study aimed to evaluate the cumulative incidence of doctor-diagnosed asthma between 31 and 46 years of age. We excluded those with current/past physician-diagnosed asthma at 31 years of age. We 
separately analysed those who reported no/current/past respiratory problems at 31 years of age, as it was hypothesised that they may interact with maternal smoke exposure on the cumulative incidence of asthma in adult offspring. Self-reported physician-diagnosed incident asthma as an outcome measure probably represents the lung function-verified asthmatic population relatively well, because a documented reversible obstruction of the lung is required for Finnish asthma drug reimbursement. Physician's reports are required to include diagnostic lung function test results in addition to background information and clinical exam results. Hence, self-reported asthma at 31 years of age could reflect asthma-like symptoms without diagnostic asthma and/or beginning asthma. Interestingly, we detected an interaction between past wheeze (before 31 years of age), gestational smoke exposure and cumulative incidence of asthma at 46 years of age as compared with the never-wheeze group. Accordingly, the adult asthma risk associated with gestational smoke exposure was also higher in the group with self-reported non-diagnosed past asthma as compared with the group who reported never-asthma. This may reflect the fact that gestational smoke exposure is related to the offspring's early respiratory problems and that these are both associated with adult-onset asthma.

After adjustments, we also found a significant association between gestational smoke exposure and the offspring's $\mathrm{FEV}_{1} / \mathrm{FVC}$ ratio at 31 years of age, reflecting the fact that the objective findings were similar to the questionnaire results. Previous studies have indicated that women may stop, reduce and sometimes restart smoking during pregnancy, which is a reflection of nicotine dependence and which also makes the measurement of maternal smoking difficult in epidemiological studies [2, 50].

Our study also has some limitations that need to be considered. These include the fact that no objective smoking markers were measured from pregnant mothers. A further shortcoming is that we cannot separate the direct effects of maternal smoking on the developing child from shared genetic and postnatal lifestyle influences on adult asthma risk, including passive smoking in the childhood house, as well as other ETS. It is likely that women who still smoked 3 months before delivery, continued to smoke during the offspring's childhood. The extent to which maternal smoking in pregnancy has persisting effects on asthma development in later life remains uncertain. We acknowledge that residual confounding may remain after adjustment for the offspring's smoking status at 31 years of age, as smoking status is likely to change over time. In this study, asthma diagnoses in early life were missing. In addition, we did not ask about the time of onset and the duration of previous self-reported asthma or wheeze. We only know that the onset and remission of these symptoms occurred before 30 years of age. We acknowledge the fact that despite our efforts in the definition of physician-diagnosed asthma, which was lung function test-confirmed in our population, slight misclassification may exist. Our findings regarding the interaction between the RUNX1 haplotype and gestational smoke exposure in the cumulative incidence of asthma between 31 and 46 years of age, were based on a small sample size; thus, further studies on genomeenvironmental interaction and on gene expression level are mandatory.

Overall, our study was able to demonstrate new evidence of an association between gestational smoke exposure and the incidence of asthma, as well as an association between gestational smoke exposure and lung function in middle-aged offspring. Maternal smoking during pregnancy was associated with the cumulative incidence of asthma in offspring between 31 and 46 years of age. The association was accentuated in offspring who reported at 31 years of age as having past respiratory problems and/or who had haplotype rs11702779-AA. In addition, a reduction in the $\mathrm{FEV}_{1} / \mathrm{FVC}$ ratio was observed at 31 years of age in the offspring with gestational smoke exposure. These results could reflect early vulnerability of the offspring's airways to ETS and its putative long-term effects. Our findings strengthen the previous hypothesis that maternal smoking has wide and long-lasting consequences for the airway health of offspring, and highlights the need to encourage women of childbearing age to permanently cease smoking.

Author contributions: All authors participated on the planning and conception of the study and the analytical strategy, B. Xu, S. Toppila-Salmi and A.T. Luukkainen wrote the manuscript. S. Toppila-Salmi, B. Xu, A.T. Luukkainen, K. Dhaygude, J. Lampi and J. Pekkanen have performed data management and analyses. All authors have performed critical review of the manuscript. The corresponding author affirms that the manuscript is an honest, accurate, and transparent account of the study being reported; that no important aspects of the study have been omitted; and that any discrepancies from the study as planned have been explained.

Conflict of interest: S. Toppila-Salmi has been a consultant for Mylan Laboratories Ltd, ERT Ltd and Roche Products Ltd, outside the submitted work. A.T. Luukkainen has nothing to disclose. B. Xu has nothing to disclose. J. Lampi has nothing to disclose. J. Auvinen has nothing to disclose. K. Dhaygude has nothing to disclose. M-R. Järvelin has nothing to disclose. J. Pekkanen has nothing to disclose.

Support statement: The study was supported in part by research grants from the Finnish Society of Allergology and Immunology, the Jane and Aatos Erkko Foundation, the Finnish Cultural Foundation, State funding for university-level health research (TYH2018103, TYH2019322), Paulo Foundation, the Tampere Tuberculosis Foundation, the Väinö and Laina Kivi Foundation. Funding information for this article has been deposited with the Crossref Funder Registry. 


\section{References}

1 Lange S, Probst C, Rehm J, et al. National, regional, and global prevalence of smoking during pregnancy in the general population: a systematic review and meta-analysis. Lancet Glob Health 2018; 6: e769-e776.

2 Gray R, Bonellie SR, Chalmers J, et al. Contribution of smoking during pregnancy to inequalities in stillbirth and infant death in Scotland 1994-2003: retrospective population based study using hospital maternity records. BMJ 2009; 339: b3754.

3 Zacharasiewicz A. Maternal smoking in pregnancy and its influence on childhood asthma. ERJ Open Res 2016; 2: 00042-2016.

4 Dempsey DA, Benowitz NL. Risks and benefits of nicotine to aid smoking cessation in pregnancy. Drug Saf 2001; 24: $277-322$.

5 Hylkema MN, Blacquiere MJ. Intrauterine effects of maternal smoking on sensitization, asthma, and chronic obstructive pulmonary disease. Proc Am Thorac Soc 2009; 6: 660-662.

6 Strachan DP, Cook DG. Health effects of passive smoking. 6. Parental smoking and childhood asthma: longitudinal and case-control studies. Thorax 1998; 53: 204-212.

7 Aspberg S, Dahlquist G, Kahan T, et al. Confirmed association between neonatal phototherapy or neonatal icterus and risk of childhood asthma. Pediatr Allergy Immunol 2010; 21: e733-e739.

8 Burke H, Leonardi-Bee J, Hashim A, et al. Prenatal and passive smoke exposure and incidence of asthma and wheeze: systematic review and meta-analysis. Pediatrics 2012; 129: 735-744.

9 Neuman A, Hohmann C, Orsini N, et al. Maternal smoking in pregnancy and asthma in preschool children: a pooled analysis of eight birth cohorts. Am J Respir Crit Care Med 2012; 186: 1037-1043.

10 Hollams EM, de Klerk NH, Holt PG, et al. Persistent effects of maternal smoking during pregnancy on lung function and asthma in adolescents. Am J Respir Crit Care Med 2014; 189: 401-407.

11 Lajunen K, Kalliola S, Kotaniemi-Syrjanen A, et al. Environmental tobacco smoke affects lung function of preschoolers with asthma even after a decade. Am J Respir Crit Care Med 2019; 199: 534-536.

12 Svanes C, Omenaas E, Jarvis D, et al. Parental smoking in childhood and adult obstructive lung disease: results from the European Community Respiratory Health Survey. Thorax 2004; 59: 295-302.

13 Grabenhenrich LB, Gough H, Reich A, et al. Early-life determinants of asthma from birth to age 20 years: a German birth cohort study. I Allergy Clin Immunol 2014; 133: 979-988.

14 Laerum BN, Svanes C, Wentzel-Larsen T, et al. Young maternal age at delivery is associated with asthma in adult offspring. Respir Med 2007; 101: 1431-1438.

15 Skorge TD, Eagan TM, Eide GE, et al. The adult incidence of asthma and respiratory symptoms by passive smoking in uterus or in childhood. Am J Respir Crit Care Med 2005; 172: 61-66.

16 Wang C, Salam MT, Islam T, et al. Effects of in utero and childhood tobacco smoke exposure and beta2-adrenergic receptor genotype on childhood asthma and wheezing. Pediatrics 2008; 122: e107-e114.

17 Haley KJ, Lasky-Su J, Manoli SE, et al. RUNX transcription factors: association with pediatric asthma and modulated by maternal smoking. Am J Physiol Lung Cell Mol Physiol 2011; 301: L693-L701.

18 Marinho S, Custovic A, Marsden P, et al. 17q12-21 variants are associated with asthma and interact with active smoking in an adult population from the United Kingdom. Ann Allergy Asthma Immunol 2012; 108: $402-411$.

19 Gilliland FD, Li YF, Dubeau L, et al. Effects of glutathione S-transferase M1, maternal smoking during pregnancy, and environmental tobacco smoke on asthma and wheezing in children. Am J Respir Crit Care Med 2002; 166: 457-463.

20 Miyake Y, Tanaka K, Arakawa M. IL3 rs40401 polymorphism and interaction with smoking in risk of asthma in Japanese women: the Kyushu Okinawa Maternal and Child Health study. Scand J Immunol 2014; 79: 410-414.

21 Reddy P, Naidoo RN, Robins TG, et al. GSTM1, GSTP1, and NQO1 polymorphisms and susceptibility to atopy and airway hyperresponsiveness among South African schoolchildren. Lung 2010; 188: 409-414.

22 Vonk JM, Scholtens S, Postma DS, et al. Adult onset asthma and interaction between genes and active tobacco smoking: the GABRIEL consortium. PLoS One 2017; 12: e0172716.

23 Rantakallio P. Groups at risk in low birth weight infants and perinatal mortality. Acta Paediatr Scand 1969; 193: Suppl. 193, 1.

24 Xu B, Jarvelin MR, Pekkanen J. Body build and atopy. J Allergy Clin Immunol 2000; 105: 393-394.

25 Sell L, Bultmann U, Rugulies R, et al. Predicting long-term sickness absence and early retirement pension from self-reported work ability. Int Arch Occup Environ Health 2009; 82: 1133-1138.

26 Tuomi K, Huuhtanen P, Nykyri E, et al. Promotion of work ability, the quality of work and retirement. Occup Med (Lond) 2001; 51: 318-324.

27 Lampi J, Koskela H, Hartikainen AL, et al. Farm environment during infancy and lung function at the age of 31: a prospective birth cohort study in Finland. BMJ Open 2015; 5: e007350.

28 Schierding W, Antony J, Karhunen V, et al. GWAS on prolonged gestation (post-term birth): analysis of successive Finnish birth cohorts. J Med Genet 2018; 55: 55-63.

29 Durinck S, Spellman PT, Birney E, et al. Mapping identifiers for the integration of genomic datasets with the R/ Bioconductor package biomaRt. Nat Protoc 2009; 4: 1184-1191.

30 Wang K, Li M, Hakonarson H. ANNOVAR: functional annotation of genetic variants from high-throughput sequencing data. Nucleic Acids Res 2010; 38: e164.

31 Wilusz CJ, Wilusz J. Lsm proteins and Hfq: life at the 3' end. RNA Biol 2013; 10: 592-601.

32 Tharun S. Roles of eukaryotic Lsm proteins in the regulation of mRNA function. Int Rev Cell Mol Biol 2009; 272: 149-189.

33 Magnus MC, Haberg SE, Karlstad O, et al. Grandmother's smoking when pregnant with the mother and asthma in the grandchild: the Norwegian Mother and Child Cohort Study. Thorax 2015; 70: 237-243.

34 Lodge CJ, Braback L, Lowe AJ, et al. Grandmaternal smoking increases asthma risk in grandchildren: a nationwide Swedish cohort. Clin Exp Allergy 2018; 48: 167-174.

35 Kalliola S, Pelkonen AS, Malmberg LP, et al. Maternal smoking affects lung function and airway inflammation in young children with multiple-trigger wheeze. J Allergy Clin Immunol 2013; 131: 730-735.

36 Tai A, Tran H, Roberts M, et al. Outcomes of childhood asthma to the age of 50 years. J Allergy Clin Immunol 2014; 133: 1572-1578. 
37 Lynch SJ, Sears MR, Hancox RJ. Thumb-sucking, nail-biting, and atopic sensitization, asthma, and hay fever. Pediatrics 2016; 138: e20160443.

38 Jaakkola MS, Piipari R, Jaakkola N, et al. Environmental tobacco smoke and adult-onset asthma: a population-based incident case-control study. Am J Public Health 2003; 93: 2055-2060.

39 Perret JL, Walters H, Johns D, et al. Mother's smoking and complex lung function of offspring in middle age: a cohort study from childhood. Respirology 2016; 21: 911-919.

40 Dratva J, Zemp E, Dharmage SC, et al. Early life origins of lung ageing: early life exposures and lung function decline in adulthood in two European cohorts aged 28-73 years. PLoS One 2016; 11: e0145127.

41 Accordini S, Calciano L, Johannessen A, et al. A three-generation study on the association of tobacco smoking with asthma. Int J Epidemiol 2018; 47: 1106-1117.

42 Upton MN, Smith GD, McConnachie A, et al. Maternal and personal cigarette smoking synergize to increase airflow limitation in adults. Am J Respir Crit Care Med 2004; 169: 479-487.

43 Brown SW, Liu B, Taioli E. The relationship between tobacco smoke exposure and airflow obstruction in US children: analysis of the national health and nutrition examination survey (2007-2012). Chest 2018; 153: 630-637.

44 Colilla S, Nicolae D, Pluzhnikov A, et al. Evidence for gene-environment interactions in a linkage study of asthma and smoking exposure. J Allergy Clin Immunol 2003; 111: 840-846.

45 Flodin U, Jonsson P, Ziegler J, et al. An epidemiologic study of bronchial asthma and smoking. Epidemiology 1995; 6: 503-505.

46 Vesterinen E, Kaprio J, Koskenvuo M. Prospective study of asthma in relation to smoking habits among 14,729 adults. Thorax 1988; 43: 534-539.

47 Toren K, Hermansson BA. Incidence rate of adult-onset asthma in relation to age, sex, atopy and smoking: a Swedish population-based study of 15813 adults. Int J Tuberc Lung Dis 1999; 3: 192-197.

48 Leonardi-Bee J, Jere ML, Britton J. Exposure to parental and sibling smoking and the risk of smoking uptake in childhood and adolescence: a systematic review and meta-analysis. Thorax 2011; 66: 847-855.

49 Weitzman M, Gortmaker S, Walker DK, et al. Maternal smoking and childhood asthma. Pediatrics 1990; 85: 505-511.

50 Pickett KE, Rathouz PJ, Kasza K, et al. Self-reported smoking, cotinine levels, and patterns of smoking in pregnancy. Paediatr Perinat Epidemiol 2005; 19: 368-376. 